\title{
Inanspruchnahme von Angeboten der Extremismusprävention
}

\section{Ergebnisse einer bevölkerungsrepräsentativen Studie}

\author{
Marc Allroggen ${ }^{1}$ Thea Rau ${ }^{1}$ \\ Eingegangen: 19. April 2021 / Angenommen: 8. Juni 2021 / Online publiziert: 21. Juli 2021 \\ (c) Der/die Autor(en) 2021
}

\section{Zusammenfassung}

Maßnahmen der Extremismusprävention in Deutschland erfolgen durch eine Vielzahl von staatlichen und zivilgesellschaftlichen Akteuren. Bislang liegen jedoch keine systematischen Untersuchungen vor, wie diese in der Allgemeinbevölkerung bekannt sind und angenommen werden. Die vorliegende bevölkerungsrepräsentative Studie geht der Frage nach, wie häufig die Allgemeinbevölkerung Rat und Hilfe zum Thema Extremismus gesucht hat, und bei welchen Anlaufstellen. Sofern bislang kein Bedarf an Beratung bestand, wurden präferierte Anlaufstellen im Falle eines Beratungsbedarfs erfragt. In beiden Fällen werden eher unspezifische Anlaufstellen wie Erziehungsberatungsstellen gewählt, aber auch Sicherheitsbehörden. Weniger häufig werden spezialisierte Fachberatungsstellen genannt. Es finden sich zudem Unterschiede bei den bevorzugten Unterstützungsangeboten in Bezug auf Alter, Geschlecht, politische Orientierung sowie Haushaltseinkommen und Bildungsstatuts. Die Studie zeigt, dass Fachberatungsstellen noch stärker in das Bewusstsein der Öffentlichkeit treten müssen, um im Bedarfsfall angesprochen werden zu können. Zudem gibt sie Hinweise darauf, dass es eine gute Vernetzung verschiedener Anlaufstellen benötigt, um Menschen mit Beratungsbedarf situationsangemessen beraten zu können.

Schlüsselwörter Radikalisierung · Fachberatungsstellen · Deradikalisierung · Vernetzung · Disengagement

\section{Utilization of extremism prevention services}

Results of a population representative study

\begin{abstract}
Measures to prevent extremism in Germany are provided by a large number of state and civil society actors. So far, however, there are no systematic studies addressing the acceptance and awareness of these actors in the general population. This representative study addressed the question of how often and at which contact points the general population sought advice and help on the topic of extremism. If there has been no need for advice so far, it was asked where advice would be sought if it were needed. It was found that in both cases non-specific contact points, such as child guidance agencies or security services tended to be chosen. Specialized counseling centers were mentioned less frequently. There were also differences in the preferred support with respect to age, gender, political orientation as well as household income and educational status. The study showed that specialized counseling centers need to be more widely recognized by the public so that they can be approached in case the need arises. It also indicated that there is a need for good networking between different contact points in order to be able to appropriately advise people in need of counseling depending on the situation.
\end{abstract}

Keywords Radicalization $\cdot$ Counselling centers $\cdot$ Deradicalisation $\cdot$ Network $\cdot$ Disengagement

PD Dr. Marc Allroggen

marc.allroggen@uniklinik-ulm.de
Klinik für Kinder- und Jugendpsychiatrie/Psychotherapie, Universitätsklinikum Ulm, Steinhövelstraße 5, 89075 Ulm, Deutschland 


\section{Einleitung}

Prävention von extremistisch motivierter Kriminalität stellt eine gesamtgesellschaftliche Aufgabe dar, die in Deutschland durch einige spezifische Aspekte geprägt ist. Hierzu gehören vor dem Hintergrund der föderalen Struktur die Verankerung vieler Präventionsangebote auf kommunaler Ebene, das Zusammenspiel von staatlichen und zivilgesellschaftlichen Trägern sowie ein Ansatz, der nicht nur auf extremistische Gewalt fokussiert, sondern auch Aspekte wie Demokratiefeindlichkeit und radikale Strömungen ohne Gewaltbereitschaft adressiert (Ben Slama 2020). Dadurch entstand eine breite, durchaus flächendeckende, aber unübersichtliche Präventionslandschaft (Gruber et al. 2016). Auch wenn in den letzten Jahren zunehmend versucht wird, Angebote der Extremismusprävention systematisch zu erfassen und darzustellen, liegen bislang keine Untersuchungen vor, die den möglichen Zugang der Allgemeinbevölkerung zu diesen Angeboten in den Blick genommen hat. Im Rahmen einer bevölkerungsrepräsentativen Studie soll erstmals erfasst werden, an wen sich die Allgemeinbevölkerung im Falle eines Beratungsbedarfs im Zusammenhang mit dem Thema Extremismus wenden würde.

Der jährliche Verfassungsschutzbericht des Bundesministerium des Innern, für Bau und Heimat weist deutlich auf die Notwendigkeit für gelingende Extremismusprävention hin (Bundesministerium des Innern, für Bau und Heimat 2020). Bei den durch das Bundeskriminalamt (BKA) für das Jahr 2019 registrierten 41.177 politisch motivierten Straftaten habe bei 76,4\% ein extremistischer Hintergrund vorgelegen. Präsent seien v.a. Straf- und Gewalttaten aus dem rechten Spektrum (21.290 Delikte, davon 925 Gewalttaten), während Straftaten aus dem linken Spektrum (6449 Delikte, davon 921 Gewaltdelikte) und dem der religiösen Ideologie (362 Delikte, davon 41 Gewaltdelikte) quantitativ eine eher untergeordnete Rolle spielen würden. Dunkelfeldstudien zur Häufigkeit extremistischen Verhaltens liegen aus Deutschland bislang nicht vor. Die Autoren der „MitteStudie" der Friedrich-Ebert-Stiftung gehen allerdings davon aus, dass bei 2,4\% der Bevölkerung im Erwachsenenalter eine deutliche rechtsextreme Orientierung vorliege (Zick et al. 2019). In einer für das Bundesland Niedersachsen repräsentativen Stichprobe wurden 5,5\% der Befragten als linksextrem eingeschätzt (Baier 2015). Bezüglich religiös begründetem Extremismus liegt eine Studie aus Deutschland mit ausschließlich Personen muslimischen Glaubens vor. Hier stimmten 7,6\% dem Einsatz von Gewalt zu, wenn es der islamischen Gemeinschaft diene, und $55 \%$ stimmten dem Einsatz von Gewalt zu, wenn diese der Durchsetzung oder Verbreitung des Islams diene (Brettfeld und Wetzels 2007). Aus dem Evaluationsbericht der Beratungsstelle Radikalisierung des Bundesamt für Migration und Flüchtlinge (BAMF) ist wiederum zu entnehmen, dass dort über einen
4-Jahres-Zeitraum 3000 Anfragen über die Hotline und über E-Mails im Zusammenhang mit Radikalisierung eingingen. Davon wurden 854 Fälle an Fachberatungsstellen weitervermittelt und 237 Fälle als sicherheitsrelevant eingestuft (Uhlmann 2017).

Betrachtet man die Angebote zur Extremismusprävention in Deutschland, so ergibt sich das Bild einer ausgesprochen heterogenen Beratungslandschaft (Lützinger und Gruber 2017; Kober 2017). Aufgrund der Auswertung von 1642 Präventionsangeboten kommen Lützinger et al. (2020) zu dem Schluss, dass der größte Teil der Präventionsmaßnahmen (85\%) sich an Personen ohne Anzeichen einer Radikalisierung richte. Hierzu gehören universell-präventive Maßnahmen mit dem Fokus Demokratieförderung, Aufklärung/Information der Bevölkerung, aber auch Beratung von Fachkräften. Bereits deutlich weniger Angebote finden sich im Bereich der selektiven Prävention (47\%), bei der primär Risikogruppen für radikale Entwicklungen adressiert werden. Ein gutes Drittel der Präventionsmaßnahmen (35\%) seien der indizierten Prävention zuzuordnen, umfassen also die Arbeit mit (potenziellen) Aussteigern aus der extremistischen Szene sowie die Beratung und Unterstützung von Angehörigen. In der Praxis ist dabei eine Trennung zwischen selektiver und indizierter Prävention häufig schwierig. So adressiert selektive Prävention definitionsgemäß Risikogruppen, die noch keine Anzeichen für eine Radikalisierung zeigen. In Ermangelung an validen und aussagekräftigen Risikomarkern, bei welchen Personen tatsächlich ein erhöhtes Risiko für eine Radikalisierung besteht, werden als selektive Prävention häufig auch Maßnahmen für Personen mit ersten Radikalisierungstendenzen gewertet. Damit überschneidet sich die selektive und die indizierte Extremismusprävention, die per Definition radikalisierte Personen adressieren sollte (Allroggen et al. 2020b). Wichtige Akteure für die indizierte Prävention sind insbesondere spezialisierte Fachberatungsstellen in staatlicher und zivilgesellschaftlicher Trägerschaft sowie Sicherheitsbehörden (Ben Slama 2020). Quantitativ stellt die Beratung von Institutionen und Angehörigen gegenüber der Beratung und Begleitung von radikalisierten Personen dabei einen Schwerpunkt dar (Möller und Neuscheler 2018; Schuhmacher 2018).

Eine entscheidende Voraussetzung für einen gelingenden Beratungsprozess ist zunächst, dass Klientinnen und Klienten, unabhängig davon, ob es sich um Angehörige, radikalisierte Personen oder ratsuchende Fachkräfte handelt, Zugang zu den Fachberatungsstellen erhalten und Vertrauen für eine Beratungsanfrage fassen, was nicht immer gelingt. Zwar wurde aufgrund der Brisanz des Themas eine Hotline beim Bundesamt für Verfassungsschutz eingerichtet, die aber aufgrund mangelnder Nachfrage aus der Bevölkerung bald wieder eingestellt werden musste (Handle et al. 2020). In den letzten Jahren haben einige Fachberatungsstellen aus diesem Grund ihre Zugangswege evaluiert, 
Tab. 1 Demografische Angaben der Gesamtstichprobe, der Personen mit früherem Beratungsbedarf in Bezug auf Extremismus und der Personen ohne bisherigen Beratungsbedarf, Anzahl $n(\%)$

\begin{tabular}{|c|c|c|c|}
\hline & $\begin{array}{l}\text { Gesamtstichprobe } \\
(n=2503)\end{array}$ & $\begin{array}{l}\text { Personen mit Beratungsbedarf } \\
(n=38)\end{array}$ & $\begin{array}{l}\text { Personen ohne Beratungsbedarf } \\
(n=2465)\end{array}$ \\
\hline Männlich & $1247(49,8 \%)$ & $18(47,4 \%)$ & $1229(49,9 \%)$ \\
\hline Weiblich & $1256(50,2 \%)$ & $20(52,6 \%)$ & $1236(50,1 \%)$ \\
\hline Alter $<25$ Jahre & $254(10,1 \%)$ & $10(26,3 \%)$ & $244(9,9 \%)$ \\
\hline Alter $>25$ Jahre & $2246(89,7 \%)$ & $28(73,7 \%)$ & $2218(90,0 \%)$ \\
\hline Migrationshintergrund & $314(12,5 \%)$ & $8(21,1 \%)$ & $306(12,4 \%)$ \\
\hline $\begin{array}{l}\text { Kein Migrationshinter- } \\
\text { grund }\end{array}$ & $2189(87,5 \%)$ & $30(78,9 \%)$ & $2159(87,6 \%)$ \\
\hline \multicolumn{4}{|l|}{ Schulabschluss } \\
\hline Kein Abschluss & $52(2,1 \%)$ & $4(10,5 \%)$ & $48(1,9 \%)$ \\
\hline Haupt-/Volksschule & $702(28,0 \%)$ & $6(15,8 \%)$ & $696(28,2 \%)$ \\
\hline Mittlere Reife & $1112(44,4 \%)$ & $11(28,9 \%)$ & $1101(44,7 \%)$ \\
\hline Hochschulreife & $340(13,6 \%)$ & $9(23,7 \%)$ & $331(13,4 \%)$ \\
\hline Studium & $258(10,3 \%)$ & $8(21,1 \%)$ & $250(10,1 \%)$ \\
\hline Aktuell Schüler & $37(1,5 \%)$ & 0 & $37(1,5 \%)$ \\
\hline \multicolumn{4}{|l|}{ Haushaltseinkommen } \\
\hline$<1250 €$ & $316(12,6 \%)$ & $7(18,4 \%)$ & $309(12,5 \%)$ \\
\hline $1250-2500 €$ & $1056(42,2 \%)$ & $15(39,5 \%)$ & $1041(42,2 \%)$ \\
\hline$>2500 €$ & $1101(44,0 \%)$ & $16(42,1 \%)$ & $1085(44,0 \%)$ \\
\hline \multicolumn{4}{|l|}{ Politische Orientierung } \\
\hline Links & $265(10,6 \%)$ & $7(18,4 \%)$ & $258(10,5 \%)$ \\
\hline Rechts & $163(6,5 \%)$ & $4(10,5 \%)$ & $159(6,5 \%)$ \\
\hline
\end{tabular}

beispielweise die Hamburger „Fach- und Beratungsstelle für religiös begründete Radikalisierung - Legato“, wobei fast die Hälfe der Beratungsfälle ( $48 \%$ ) auf direktem Wege die Beratungsstelle aufgesucht haben wollen, was auf einen hohen Bekanntheitsgrad von Legato zurückgeführt wurde. In seltenen Fällen erfolgte der Zugang von Ratsuchenden über die Sicherheitsbehörden (Schuhmacher 2018). Auch bei Beratungsstellen im Bereich des Rechtsextremismus scheinen unmittelbare Zugänge zu Beratungsstellen gut zu funktionieren, wobei ein Bedarf an der Ausgestaltung jugendaffiner Zugangswege, z. B. über Facebook oder Flyer mit QR-Code, zur Ergänzung schon vorhandener virtueller Zugangswege konstatiert wird (Möller und Neuscheler 2018).

Obwohl diese Untersuchungen erste Hinweise darauf geben können, wie Ratsuchende erreicht werden können, fehlen bislang systematische Untersuchungen zu Zugangswegen zur Fachberatung. Diese zu analysieren, ist aber entscheidend, um strategische Kooperationspartner für eine Vernetzung hinsichtlich der Extremismusprävention auszuwählen (Bundesministerium für Familie, Senioren, Frauen und Jugend und Bundesministerium des Inneren 2016).

Ziel der vorliegenden Studie ist es daher, anhand einer bevölkerungsrepräsentativen Stichprobe zu erfassen, an wen sich die Befragten wenden würden, wenn sie Unterstützung zum Thema Radikalisierung benötigen würden, oder an wen sie sich gewandt haben, falls bereits ein Beratungs- und Hilfebedarf in der Vergangenheit bestand. Die Untersuchung soll dabei auch klären, ob neben den bekannten Akteuren im Feld wie Fachberatungsstellen oder Sicherheitsbehörden auch andere Institutionen und Berufsgruppen eine relevante Rolle bei der indizierten Extremismusprävention einnehmen können.

\section{Methodik}

\section{Rekrutierung und Stichprobe}

Durch ein demografisches Institut (Markt- und Sozialforschungsinstitut-USUMA, Berlin) wurde nach dem Random-Route-Verfahren mit Startadressenvorgabe eine in Bezug auf Alter, Geschlecht, Bildung und Wohnregion repräsentative Stichprobe der deutschen Bevölkerung ausgewählt. In den dementsprechend ausgewählten 5668 Haushalten wurden Interviewpartner zufällig nach einem sog. Schwedenschlüssel bestimmt. Voraussetzung war dabei die Erfüllung der Einschlusskriterien (Mindestalter 14 Jahre, ausreichende Deutschkenntnisse). Insgesamt 2522 Interviews konnten geführt werden. Ein/e Studienmitarbeiter*in klärte über die Studie auf, holte die Einwilligung zur Teilnahme ein und blieb ansprechbar, bis der/die Teilnehmende die Fragebogen ausgefüllt hatte. Bei der Information über die Studie nannten die Studienmitarbeitenden keinen kon- 
Tab. 2 Häufigkeit der genannten Anlaufstellen von Personen, bei denen bereits Unterstützungsbedarf bestand, und Personen, bei denen bislang noch kein Beratungsbedarf vorlag. Mehrfachnennungen möglich

\begin{tabular}{lll}
\hline & $\begin{array}{l}\text { Hilfe wurde gesucht bei ... } \\
\text { Anzahl } n \text { (absolute Prozent von 38) }\end{array}$ & $\begin{array}{l}\text { Hilfe würde gesucht werden bei ... } \\
\text { Anzahl } n \text { (absolute Prozent von 2465) }\end{array}$ \\
\hline Erziehungs- und Familienberatungsstelle & $16(42,1 \%)$ & $329(13,3 \%)$ \\
Fachberatung für Radikalisierung & $1(2,6 \%)$ & $322(13,1 \%)$ \\
Polizei, Landeskriminalämter, Bundeskriminalamt & $7(18,4 \%)$ & $986(40,0 \%)$ \\
Arzt/Psychologe/Psychotherapeut & $4(10,5 \%)$ & $354(14,4 \%)$ \\
Behörden (z. B. BAMF, Verfassungsschutz) & 0 & $147(6,0 \%)$ \\
Niemand & $4(10,5 \%)$ & $399(16,2 \%)$ \\
Sonstige & $10(26,3 \%)$ & $40(1,6 \%)$ \\
Keine Angabe & 0 & $48(1,9 \%)$ \\
\hline
\end{tabular}

kreten Themenbereich, sondern informierten darüber, dass es sich um eine Studie zum Thema psychische Gesundheit und Wohlbefinden handele. In der Untersuchung wurden insgesamt 119 Fragen zu unterschiedlichen Themen wie psychischen Belastungen, Gewalterfahrungen und zur politischen Einstellung gestellt. Ein positives Votum durch die Ethikkommission der Universität Leipzig liegt vor.

Von den 2522 durchgeführten Interviews konnten 2503 ausgewertet werden. Das Durchschnittsalter der Teilnehmenden lag bei 49,52 Jahren $( \pm 17,51), 50,2 \%(n=1265)$ waren weiblich. Weitere Details zur Stichprobe können der Tab. 1 im Ergebnisteil entnommen werden.

\section{Fragestellung und Auswertung}

Den Studienteilnehmenden wurde zunächst die folgende Frage gestellt: „Benötigten Sie schon mal einen Rat oder Hilfe zu dem Thema Extremismus, z.B. wegen der Radikalisierung eines Familienmitglieds, eines Freundes oder für sich selber?" Wurde diese Frage bejaht, wurde konkret gefragt „An wen haben Sie sich gewandt?“ und eine Liste mit den folgenden Auswahlmöglichkeiten vorgelegt, wobei Mehrfachnennungen möglich waren: Erziehungsund Familienberatungsstelle; Fachberatung für Radikalisierung; Polizei, Landeskriminalämter, Bundeskriminalamt; Arzt/Psychologe/Psychotherapeut; Behörden (z. B. BAMF, Verfassungsschutz); an niemanden. Zudem bestand die Möglichkeit, sonstige Institutionen/Personen als Freitext zu benennen.

Wurde die Eingangsfrage verneint, so wurde die folgende Frage gestellt: „Bei welcher Stelle würden Sie im Falle eines Beratungsbedarfs zuerst Hilfe suchen?" und die oben genannte Auswahlliste angeboten. Auch hier waren Mehrfachnennungen möglich. Ergänzend wurde bei dieser Frage untersucht, ob es Unterschiede im Antwortverhalten in Bezug auf das Geschlecht (männlich/weiblich), Alter (jünger/ älter 25 Jahre), Bildungsstand, Haushaltseinkommen, Migrationshintergrund (mindestens ein Elternteil wurde außerhalb Deutschlands geboren) sowie politische Orientierung gab. Politische Orientierung wurde erfasst mittels Selbsteinschätzung anhand einer 11-stufigen Skala von 1: „links“ bis 11: „rechts“. Als „links“ wurden alle Personen gewertet, die Angaben von 1-3 machten, als „rechts“ alle Personen, die Angaben von 9-11 machten.

Die Beantwortung der Hauptfragestellung erfolgte deskriptiv mittels Berechnung der Häufigkeit der Angaben. Gruppenunterschiede wurden mittels Pearson-Chi'2-Quadrat-Test berechnet, mit anschließender Post-hoc-Analyse auf einem Signifikanzniveau von $p<0,05$.

\section{Ergebnisse}

Von den Befragten gaben 38 Personen $(1,5 \%)$ an, schon einmal Hilfe oder Rat zum Thema Extremismus benötigt zu haben, die übrigen 2465 Befragten $(98,5 \%)$ verneinten dies (Tab. 1).

Die meisten Personen, die bereits einen Beratungsbedarf hatten, wandten sich an Erziehungs- und Familienberatungsstellen $(42,1 \% ; n=16)$ oder an Sicherheitsbehörden $(18,4 \% ; n=7)$ und Heilberufe bzw. Psychologen $(10,5 \%$; $n=4)$. An niemanden hatten sich 4 Personen gewandt (Tab. 2). Unter „Sonstige“ wurden v.a. Freund/-e $(n=7)$ genannt, sowie „Mutter“ $(n=1)$, ,Lehrer“ $(n=1)$, ,Inter-

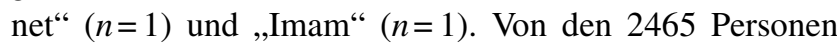
(98,5\%), die bislang noch keinen Beratungsbedarf oder Unterstützung benötigten, würden sich die meisten Befragten an Sicherheitsbehörden $(40,0 \%, n=986)$ wenden (Tab. 2). Die Anlaufstellen Erziehungs- und Familienberatungsstellen $(13,3 \%, n=329)$, Fachberatung für Radikalisierung $(13,1 \%, n=322)$ sowie Arzt/Psychologe/Psychotherapeut $(14,4 \%, n=354)$ wurden in etwa gleich häufig benannt. An niemanden würden sich 399 Personen (16,2\%) wenden (Tab. 2). Unter „Sonstige“ wurden v.a. Freunde/Partner $(n=19)$ und Familienmitglieder genannt $(n=15)$, sowie das „Internet“ ( $n=7)$ und „Pfarrer“ $(n=1)$.

Gruppenunterschiede (signifikante Unterschiede in der Post-hoc-Analyse werden mit hochgestellten Buchstaben 
gekennzeichnet) zeigen sich für den Fall eines Beratungsbedarfs bei der intendierten Inanspruchnahme von Erziehungs- und Familienberatungsstelle in Bezug auf das Geschlecht (Frauen 15,7\%, Männer 11,0\%, $\mathrm{Chi}^{2} 11,827$, $p=0,001)$. Bei der möglichen Inanspruchnahme einer Fachberatung für Radikalisierung fanden sich Unterschiede in Bezug auf das Haushaltseinkommen (Haushaltseinkommen $<1250 € 11,7 \%$, Haushaltseinkommen 1250-2500€ $10,9 \%$ a Haushaltseinkommen $>2500 € 15,7 \%$, $\mathrm{Chi}^{2}$ $11,465, p=0,003)$ sowie in Bezug auf das Bildungsniveau (kein Abschluss 4,2\%, Hauptschule 10,2\% , mittlere Reife $12,1 \%^{\mathrm{a}}$, Hochschulreife $17,5 \%$, Studium $20,8 \%^{\mathrm{ac}}$, aktuell Schüler 16,2\%, $\mathrm{Chi}^{2}$ 28,580, $\left.p=0,000\right)$. Bezüglich der Sicherheitsbehörden (Polizei, Landeskriminalämter, Bundeskriminalamt) finden sich Gruppenunterschiede hinsichtlich des Alters (Alter <25 Jahre 29,1\%, Alter $>25$ Jahre 41,2\%, Chi $^{2}$ 13,336, $p=0,000$ ), der politischen Orientierung (links 33,3\%, rechts $43,4 \%, \mathrm{Chi}^{2}$ 4,265, $p=0,039$ ) sowie des Bildungsstands (kein Abschluss $50,0 \%$, Hauptschule 37,9\%, mittlere Reife 43,5\%a, Hochschulreife $38,7 \%$, Studium $32,8 \%$ a aktuell Schüler $24,3 \%$, $\left.\mathrm{Chi}^{2} 18,306, p=0,003\right)$. Angehörige der Gesundheitsberufe (Arzt/Psychologe/Psychotherapeut) würden eher von jüngeren Personen (Alter $<25$ Jahre 18,9\%, Alter $>25$ Jahre $\left.13,9 \%, \mathrm{Chi}^{2} 4,403, p=0,039\right)$ und von Personen mit eher linker Orientierung (links $15,1 \%$, rechts $8,2 \%$, $\mathrm{Chi}^{2} 4,341$, $p=0,037$ ) angefragt werden. Bezüglich der Inanspruchnahme von Behörden (z.B. BAMF, Verfassungsschutz) ergaben sich keine signifikanten Gruppenunterschiede. Männer würden sich häufiger „niemandem“ anvertrauen (Männer $18,8 \%$, Frauen 13,6\%, $\mathrm{Chi}^{2} 12,299, p=0,000$ ), zudem fanden sich in der Kategorie „niemand“ auch Gruppenunterschiede bezüglich des Einkommens (Haushaltseinkommen $<1250 € 21,7 \%$ a , Haushaltseinkommen 1250-2500€ $18,6 \%$ b Haushaltseinkommen $>2500 € 12,3 \%$ ab $\mathrm{Chi}^{2}$ 23,834, $p=0,000$ ) und des Bildungsabschlusses (kein Abschluss $10,4 \%$, Hauptschule $22,3 \%$ bac , mittlere Reife $14,5 \%^{c}$, Hochschulreife $13,0 \%$, Studium $12,0 \%$ a aktuell Schüler 13,5\%, $\left.\mathrm{Chi}^{2} 28,340, p=0,000\right)$. Bei keiner der vorgegebenen Antwortkategorien ergaben sich Unterschiede in Bezug auf einen Migrationshintergrund.

\section{Diskussion}

Die vorliegende Studie ist nach Kenntnis der Autoren die erste bevölkerungsrepräsentative Studie, die untersucht, an wen sich Personen mit einem Beratungsbedarf in Bezug auf Extremismus wenden würden bzw. bereits gewandt haben. Sie kann wertvolle Erkenntnisse zu Zugangswegen von Ratsuchenden liefern und auf Vernetzungsbedarfe von Angeboten hinweisen, um die Zugänge zu entsprechenden Fachberatungen zu erleichtern.
Die Ergebnisse zeigen, dass etwa 1,5\% der Befragten bereits Beratungsbedarf zum Thema Extremismus hatten. Vor allem jüngere Personen und Personen mit Migrationshintergrund scheinen dabei etwas häufiger Beratung in Anspruch genommen zu haben. Dies deckt sich mit Studien, die ein jüngeres Alter und z.T. auch das Vorliegen eines Migrationshintergrundes als Risikofaktor für radikale Einstellungen nachweisen (Wolfowicz et al. 2019). Dass entgegen den Erwartungen etwas mehr Frauen mit dem Thema konfrontiert worden sind, liegt möglicherweise daran, dass v.a. Angehörige (z.B. Mütter) Beratung suchen (Schuhmacher 2018). Dies erklärt möglicherweise auch, warum überwiegend Angebote wie Erziehungs- und Familienberatungsstelle als Anlaufstelle gewählt wurden, was die Gefahr birgt, dass damit möglicherweise keine spezialisierte Beratung und professionelle Bewertung des Falles stattgefunden hat. Neben den niederschwelligen Anlaufstellen und persönlichen Ansprechpersonen wurden v. a. auch Sicherheitsbehörden (z. B. Polizei, Landeskriminalämter, Bundeskriminalamt) genannt, was darauf hindeuten könnte, dass es sich entweder um Fälle handelt, bei denen ein strafbares Handeln vermutet wurde, oder aber andere Ansprechpersonen nicht bekannt waren. Erstaunlich ist, dass sich niemand bzw. nur wenige Personen an Fachberatungsstellen oder Behörden gewandt haben. Dies kann ein Hinweis darauf sein, dass spezialisierte Beratungen bzw. auch zentrale deutschlandweite Hotlines zum Thema (Hotline „Radikalisierung" des BAMF) in der Allgemeinbevölkerung noch nicht hinreichend bekannt sind. Die Ergebnisse müssen jedoch aufgrund der niedrigen absoluten Fallzahlen mit Vorsicht interpretiert werden.

Ein ähnliches Bild zeigt sich allerdings bei der Frage, an wen sich Betroffene im Falle eines Beratungs- und Hilfebedarfs wenden würden. Auch hier werden Sicherheitsbehörden am häufigsten als Ansprechpartner benannt. Daneben werden auch eher unspezifische Angebote wie Erziehungsund Familienberatungsstellen oder Angehörige von Heilberufen genannt, jedoch, anders als bei den tatsächlichen Inanspruchnahmefällen, auch Fachberatungsstellen und Behörden, was möglicherweise darauf zurückzuführen ist, dass diese Stellen als Antwortmöglichkeiten bereits vorgegeben waren. So kann die Information im Fragebogen, dass es diese Stellen gibt, schon dazu geführt haben, dass diese auch als relevant im Falle eines Beratungsbedarfs bewertet wurden. Dies würde bedeuten, dass, wenn Fachberatungsstellen zum Thema Radikalisierung besser bekannt würden, diese möglicherweise auch als Ansprechperson im Ernstfall eher gewählt werden würden. Zudem gaben gut $16 \%$ der Befragten an, dass sie bei einem möglichen Beratungsbedarf niemanden kontaktieren würden, was fatale Folgen für die Betroffenen und die Gesellschaft haben könnte und ebenso dafür spricht, professionelle Beratung bekannt zu machen. 
Gleichzeitig zeigen die Ergebnisse, dass unterschiedliche Bevölkerungsgruppen auch unterschiedliche Unterstützungsangebote präferieren. So würden sich jüngere Personen und Personen, die ihre politische Orientierung eher als links bezeichnen, seltener an Sicherheitsbehörden wenden und häufiger Ärzte oder Psychotherapeuten als Ansprechpartner wählen, möglicherweise auch als Ausdruck eines Misstrauens gegenüber staatlichen Institutionen. Auch Frauen scheinen häufiger unspezifische und niederschwellige Beratungsangebote zu wählen. Interessant ist aber vor allen Dingen, dass sich neben Männern v.a. Personen mit niedrigerem Haushaltseinkommen und niedrigeren Bildungsabschlüssen im Falle eines Beratungsbedarfs eher an niemanden wenden würden. Für diesen Personenkreis ist jedoch das Risiko für die Entwicklung radikaler Einstellungen erhöht, gleichzeitig werden sie durch bestehende Angebote schwerer erreicht (Wolfowicz et al. 2019).

Zusammengenommen zeigen die Ergebnisse, dass Ratsuchende möglicherweise vielfach Ansprechpartner wählen, die nicht spezialisiert sind, die ihnen möglicherweise aber aus anderen Kontexten bekannt sind. So spielt trotz der berichteten hohen Fallzahlen der Beratungsstelle des BAMF (Uhlmann 2017) diese als primärer Ansprechpartner bei den Befragten keine nennenswerte Rolle. Auch der von Legato berichtete hohe Anteil an direktem Zugang spiegelt sich in den hier erhobenen Daten nicht wider (Schuhmacher 2018). Daraus kann geschlossen werden, dass Fachberatungsstellen bei der Allgemeinbevölkerung noch stärker publik gemacht werden sollten. Da Öffentlichkeitsarbeit und komplementäre Zugangswege zu Fachberatungsstellen jedoch eine hohe Personalkapazität erfordern (Möller und Neuscheler 2018), sind die finanziellen Voraussetzungen dafür in den Beratungsstellen vorerst zu prüfen.

In diesem Zusammenhang müssen aber auch mögliche Limitationen der Studie betrachtet werden. So wurden aufgrund des Haushaltszuganges unter Umständen Personen, die bereits Kontakt zu Fachberatungsstellen hatten oder sog. Aussteiger aus extremistischen Gruppierungen nicht erreicht, weil diese einen Zugang zu ihrer Wohnung eher ablehnen und bei einer Teilnahme möglicherweise auch Repressalien durch andere radikale Personen fürchten. Für andere wiederum mag das Thema Extremismus so abstrakt sein, dass diese sich noch keine Gedanken darüber gemacht haben, an wen sie sich gezielt wenden würden, und daher (trotz der Antwortmöglichkeiten) primär eher niederschwellige und unspezifische Optionen gewählt haben.

Dennoch lassen sich auf der Basis dieser ersten bevölkerungsrepräsentativen Stichprobe wertvolle Hinweise gewinnen und einige Herausforderungen insbesondere für die indizierte Extremismusprävention ableiten. Die Tatsache, dass viele Ratsuchende eher niederschwellige und unspezifische Beratungsangebote aufsuchten bzw. aufsuchen würden, birgt die Gefahr, dass diese mit der Fragestellung überfordert sind und Ratsuchende den Weg in eine Fachberatung nicht finden. Es ist daher zwingend notwendig, Familien- und Erziehungsberatungsstellen sowie Ärzte und Psychotherapeuten in entsprechende Netzwerke einzubinden. Für den Bereich der Heilberufe wurde beispielsweise mit Unterstützung des BAMF eine Handlungsempfehlung für Ärzte und Psychotherapeuten entwickelt, die eine Vernetzung befördern und Hinweise im Umgang mit Beratungsfällen geben soll (Allroggen et al. 2020a). Zudem ist eine Einbindung der Akteure in bestehende Präventionsnetzwerke stärker mitzudenken (Modell- und Kooperationsprojekt TRIAS, Zugang zu psychotherapeutischer Behandlung bei Deradikalisierungsprozessen, http://www. extremismus-und-psychologie.de/Projekt-TRIAS/). Ähnliche Projekte könnten auch für den Bereich der Erziehungsund Familienberatungsstellen und für weitere Anlaufstellen förderlich sein. Die Strategie der Bundesregierung zur Extremismusprävention sieht bereits eine Vernetzung von verschiedenen Akteuren vor (Bundesministerium für Familie, Senioren, Frauen und Jugend und Bundesministerium des Inneren 2016), berücksichtigt allerdings nicht Angehörige von Heilberufen.

So sollte einerseits durch Netzwerkarbeit der Weg für Betroffene zu den Fachberatungsstellen erleichtert werden, wenn sich diese zunächst an unspezifische Angebote wenden. Gleichzeitig sollten diese Akteure und Anlaufstellen aber auch stärker in den Beratungs- und Deradikalisierungsprozess eingebunden werden, um beispielsweise flankierende Probleme oder einen gleichzeitig bestehenden Bedarf der Behandlung, etwa im Falle einer psychischen Störung, abzudecken (Lützinger und Gruber 2017). Zudem könnten diese Anlaufstellen, wenn bereits ein Vertrauensverhältnis besteht, den Deradikalisierungsprozess auch unterstützend und motivierend begleiten.

Förderung Die bevölkerungsrepräsentative Studie wurde aus Mitteln des Bundesamt für Migration und Flüchtlinge (BAMF) finanziert.

Funding Open Access funding enabled and organized by Projekt DEAL.

Interessenkonflikt M. Allroggen und T. Rau geben an, dass kein Interessenkonflikt besteht.

Open Access Dieser Artikel wird unter der Creative Commons Namensnennung 4.0 International Lizenz veröffentlicht, welche die Nutzung, Vervielfältigung, Bearbeitung, Verbreitung und Wiedergabe in jeglichem Medium und Format erlaubt, sofern Sie den/die ursprünglichen Autor(en) und die Quelle ordnungsgemäß nennen, einen Link zur Creative Commons Lizenz beifügen und angeben, ob Änderungen vorgenommen wurden.

Die in diesem Artikel enthaltenen Bilder und sonstiges Drittmaterial unterliegen ebenfalls der genannten Creative Commons Lizenz, sofern sich aus der Abbildungslegende nichts anderes ergibt. Sofern das betreffende Material nicht unter der genannten Creative Commons Lizenz steht und die betreffende Handlung nicht nach gesetzlichen Vorschrif- 
ten erlaubt ist, ist für die oben aufgeführten Weiterverwendungen des Materials die Einwilligung des jeweiligen Rechteinhabers einzuholen.

Weitere Details zur Lizenz entnehmen Sie bitte der Lizenzinformation auf http://creativecommons.org/licenses/by/4.0/deed.de.

\section{Literatur}

Allroggen M, Rau T, Schmidt H, Fegert JM (2020a) Handlungsfeld „Indizierte Extremismusprävention“. In: Slama BB, Kemmesies U (Hrsg) Handbuch Extremismusprävention - Gesamtgesellschaftlich. Phänomenübergreifend. Bundeskriminalamt, Wiesbaden, S 505-522

Allroggen M, Heimgartner A, Rau T, Fegert JM (2020b) Radikalisierungsprozesse wahrnehmen - einschätzen - handeln: Grundlagenwissen für Ärzt*innen und Psychotherapeut*innen. Universitätsklinikum Ulm. https://www.uniklinikulm.de/fileadmin/default/Kliniken/Kinder-Jugendpsychiatrie/ Dokumente/Handlungsempfehlung_Radikalisierungsprozesse. pdf. Zugegriffen: 1. Apr. 2021

Baier D (2015) Sicherheit und Kriminalität in Niedersachsen. Ergebnisse einer Repräsentativbefragung. KFN: Forschungsbericht Nr. 127

Brettfeld K, Wetzels P (2007) Muslime in Deutschland. Integration, Integrationsbarrieren, Religion sowie Einstellungen zu Demokratie, Rechtsstaat und politisch-religiös motivierter Gewalt. Bundesministerium des Inneren, Berlin

Bundesministerium des Innern, für Bau und Heimat (2020) Verfassungsschutzbericht 2019. https://www.verfassungsschutz.de/de/ download-manager/_vsbericht-2019.pdf. Zugegriffen: 1. Apr. 2021

Bundesministerium für Familie, Senioren, Frauen und Jugend, Bundesministerium des Inneren (2016) Strategie der Bundesregierung zur Extremismusprävention und Demokratieförderung. https:// www.bmfsfj.de/blob/109002/5278d578ff8c59a19d4bef9fe4c034 d8/strategie-der-bundesregierung-zur-extremismuspraeventionund-demokratiefoerderung-data.pdf. Zugegriffen: 1. Apr. 2021

Gruber F, Lützinger S, Kemmesies UE (2016) Extremismusprävention in Deutschland. Erhebung und Darstellung der Präventionslandschaft. Schwerpunktdarstellung Präventionsprojekte in staatlicher Trägerschaft (2014/2015). (unter Mitarbeit von I. Klima, G. Sielaff \& S. Wick). Bundeskriminalamt, Wiesbaden

Handle J, Korn J, Mücke T (2020) Zivilgesellschaftliche Organisationen in der Tertiärprävention. Violence Prevention Network. Schriftenreihe Heft 3. https://violence-prevention-network. de/wp-content/uploads/2020/01/Violence-Prevention-NetworkSchriftenreihe-Heft-3-1.pdf. Zugegriffen: 1. Apr. 2021
Kober M (2017) Zur Evaluation von Maßnahmen der Prävention von religiöser Radikalisierung in Deutschland. J Deradicalization 11:219-257

Lützinger S, Gruber F (2017) Extremismusprävention in Deutschland - Herausforderungen und Optimierungspotential. Modulabschlussbericht. Wiesbaden: Bundeskriminalamt. https:// www.bka.de/SharedDocs/Downloads/DE/Publikationen/ Publikationsreihen/Forschungsergebnisse/2017PueG_Extremis muspraeventionInDeutschland_Herausforderung.html. Zugegriffen: 1. Apr. 2021

Lützinger S, Gruber F, Hedayt A (2020) Eine Bestandsaufnahme präventiver Angebote in Deutschland sowie ausgewählter Präventionsstrategien aus dem europäischen Ausland. In: B. Ben Slama \& U. Kemmesies (Hrsg.), Handbuch Extremismusprävention - Gesamtgesellschaftlich. Phänomenübergreifend (S. 597-626). Wiesbaden: Bundeskriminalamt.

Möller K, Neuscheler F (2018) Abschlussbericht zur Evaluation der Beratungsstelle Hessen - Religiöse Toleranz statt Extremismus. Beratungsstelle Hessen Religiöse Toleranz statt Extremismus. Esslingen. https://violence-prevention-network.de/wp-content/ uploads/2019/02/Abschlussbericht-Evaluation-BeratungsstelleHessen.pdf. Zugegriffen: 1. Apr. 2021

Schuhmacher N (2018) Evaluation der Hamburger ,Fach- und Beratungsstelle für religiös begründete Radikalisierung - Legato، (unter Mitarbeit von G. Kowol). Hamburg: Universität Hamburg, Kriminologische Sozialforschung. https://www.wiso.unihamburg.de/fachbereich-sowi/professuren/hentschel/forschung/ legato/legato-abschlussbericht-voe.pdf. Zugegriffen: 1. Apr. 2021

Slama BB (2020) Extremismusprävention aus der Perspektive eines sicherheitsbehördlichen Bedarfs. In: Slama B, Kemmesies U (Hrsg) Handbuch Extremismusprävention - Gesamtgesellschaftlich. Phänomenübergreifend. Bundeskriminalamt, Wiesbaden, S 429-449

Uhlmann M (2017) Evaluation der Beratungsstelle „Radikalisierung“. Abschlussbericht. Nürnberg: Bundesamt für Migration und Flüchtlinge. Forschungsbericht 31. https://www.bamf. de/SharedDocs/Anlagen/DE/Forschung/Forschungsberichte/ fb31-evaluation-beratungsstelle-radikalisierung.pdf? blob=publicationFile\&v=15. Zugegriffen: 1. Apr. 2021

Wolfowicz M, Litmanovitz Y, Weisburd D, Hasisi B (2019) A field-wide systematic review and meta-analysis of putative risk and protective factors for radicalization outcomes. J Quant 36(3):407-447. https://doi.org/10.1007/s10940-019-09439-4

Zick A, Küpper B, Berghan W (2019) Verlorene Mitte - Feindselige Zustände Rechtsextreme Einstellungen in Deutschland 2018/19. Dietz, Bonn 